J3eA, Journal sur l'enseignement des sciences et technologies de l'information et des systèmes, Volume 2, Hors-Série 2, 8 (2003)

DOI : http://dx.doi.org/10.1051/bib-j3ea:2003608

(C) EDP Sciences, 2003

Biopuces pour le traitement électrique paraléllisé de cellules

B. Le Pioufle*, M. Frénéa* et A. Tixier**

* BIOMIS/SATIE CNRS UMR 8029

ENS Cachan

61 avenue du président Wilson

F-94235 Cachan Cedex, France

** LIMMS CNRS Université de Tokyo

4-6-1 Komba, Meguro-ki

Tokyo, 153-8505, Japon

pioufle@bretagne.ens-cachan.fr 


\title{
Biopuces pour le traitement électrique paraléllisé de cellules
}

\author{
B. Le Pioufle, M. Frénéa, \\ BIOMIS/SATIE CNRS UMR 8029, ENS Cachan \\ 61 Av du Pt Wilson, 94235 Cachan Cedex \\ France \\ E-mail:. pioufle@bretagne.ens-cachan.fr \\ Agnès Tixier \\ LIMMSCNRS Université de Tokyo \\ 4-6-1, Komaba, Meguro-ku, Toky o, 153-8505, \\ Japon
}

\section{Résumé}

Après un bref état de l'art sur la recherche dans le domaine des biopuces cellulaires, nous présentons les travaux en cours dans notre laboratoire sur l'étude et la conception d'une biopuce pour la manipulation hautement parallélisée de cellules par champ électrique.

\section{Introduction : les biopuces cellulaires}

La technologie puce à $\mathrm{ADN}$ a montré l'impact que peuvent avoir les technologies dérivées de la microélectronique sur les sciences du vivant. Ces biopuces sont en effet des outils clef pour l'étude du génome, du transcriptome, et pour le diagnostique. L'impact socioéconomique de ce type de biopuces est potentiellement considérable. Le succès de cette technologie "biopuce à $\mathrm{ADN} »$ a conduit les chercheurs à imaginer d'autres types de biopuces : la biopuce à protéines permettant d'analyser les fonctions cellulaires par la détection directe des protéines cytoplasmiques, et enfin plus récemment la biopuce à cellules, où des cellules vivantes entières sont micromanipulées et /ou analysées.

Une biopuce à cellules est une puce électronique destinée à la micromanipulation de cellules vivantes. Le pionnier de cette thématique de recherche est probablement le Professeur Washizu (Université de Kyoto) qui a proposé en 1989 une biopuce permettant la fusion de deux cellules par électro-perméabilisation de la membrane cytoplasmique [1] .

Depuis cette invention, plusieurs catégories de biopuces à cellules ont vu le jour dans les laboratoires de recherche :

- les biopuces cellulaires parallèles : à l'image des biopuces ADN qui mettent un grand nombre d'oligonucléotide en réseau, les biopuces cellulaires parallèles mettent un grand nombre de cellules vivantes en réseau, à des fin d'analyse ou traitement simultané. Notre équipe a été une des première à proposer le principe de biopuces cellulaires parallèles.

- les biopuces séries : où les cellules sont convoyées une à une et en série par microfluidique

- les biopuces pour la manipulation et l'analyse de cellules uniques

Ces variantes structurelles de biopuces cellulaires permettent la manipulation par voie électrique, mécanique ou biochimique d'une cellule vivante unique ou d'un grand nombre de cellules vivantes à la fois, pour diverses applications : tri cellulaire, fusion cellulaire, transfert de gènes, ...

\section{Microdispositifs pour le tri cellulaire:}

Un nombre important de travaux concernent la conception de micro-dispositifs pour le tri cellulaire. Les cellules vivantes sont triées en ligne par un dispositif microfluidique série, ou en parallèle. Plusieurs modes de tris sont envisagés :

- par diélectrophorèse, en utilisant la différence de permittivité diélectrique entre la cellule et le miliau environnant. On peut citer les travaux de Fuhr (Université de Berlin) [2], ou Morgan (Glasgow) [3] qui proposent des dispositifs série de manipulation par diélectrophorèse, ou encore les travaux de M. Frénéa (BIOMIS) [4] qui propose des biopuces parallèles pour le rangement de cellules par diélectrophorèse.

- Par voie mécanique ou fluidique: une force mécanique permet de mettre en mouvement une ou plusieurs cellules. La force exténieure peut être produite par un dispositif mécanique : citons les travaux de E. Piat (LAB, Besançon) [5]. Elle peut également être produite par un mouvement fluidique: citons les travaux de A. Tixier (Université de Tokyo) [6], qui range les cellules par l'aspiration de nano-capillaires

- Par voie biochimique : une microstructuration du substrat permet de guider l'adhésion cellulaire sur des surfaces prédéfinies. On peut citer les travaux de G. Whitesides (Harvard, USA) [7], D. Ingber (Harvard Medical School),

Puces à neurones: une autre application des biopuces cellulaires, et donc de la microculture sur puce, concerne les neurones. Il s'agit en général d'exciter ou recueillir des potentiels membranaires sur neurones en culture sur la puce : travaux de Pine (Caltech) [8], Offenhauser (Glasgow) [9], ou de faire de la croissance guidée de neurones : travaux d' Offenhauser (Glasgow), Tamiya (JAIST), Griscom (LIMMS,BIOMIS) [10].

\section{Puce à cellules parallèles pour le transfert de gènes}

L'originalité de notre démarche consiste à étudier et concevoir des puces à cellules hautement parallélisées, traitant à la fois un très grand nombre de cellules vivantes 
(depuis quelques milliers jusqu'à plusieurs centaines de milliers). Dans ce cadre, un de nos axes de recherche principaux concerne l'étude et la conception de puces cellulaires parallèles pour le transfert de gènes [11]. En effet la thérapie génique est une méthode d'avenir pour le traitement des maladies génétiques, mais dont le développement est encore limité par le manque d'efficacité des methodes actuelles de transfert de gene. Pour cette raison nous travaillons sur le concept d'une biopuce capable de prendre en charge cette étape de transfection afin 1) d'analyser finement et comprendre sur cellules uniques en micro-culture les limitations physiques du transfert de gène 2) à terme augmenter le taux d'efficacité de la transfection. Le microdispositif propose est conçu pour avoir la capacité de ranger les cellules vivantes dans un réseau bi-dimensionnel de haute densité, et de transférer le gène en milieu intra-cellulaire par une microélectroporation des cellules ainsi isolées et microcultivées.

En ce qui concerne les aspects du rangement en réseau des cellules, plusieurs strategies ont été développées parallèlement et testées dans notre laboratoire: 1) l'utilisation d'une microfluidique d'aspiration des cellules (micro-capillaires micro-usinés dans le silicium) 2) en combinant l'aspiration mécanique à une capture spécifique des cellules sur le substrat, par greffage d'anticorps spécifiques (voir figure 1) 3) en appliquant une force de diélectrophorèse sur les cellules, grâce à une topologie particulière du champ électrique sur la biopuce (voir figure 2) [4].

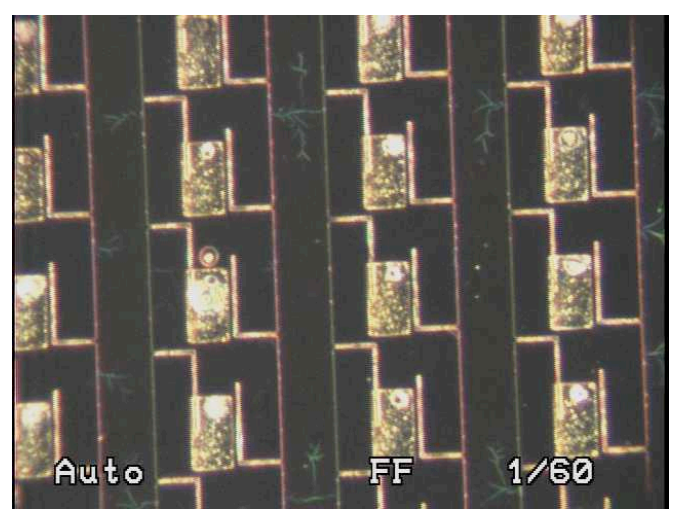

Figure 1: Biopuce cellulaire - rangement de cellules uniques en réseau par action combinée (aspiration + liaison biochimique)

SATIE/BIOMIS, Univ. Tokyo, JAIST

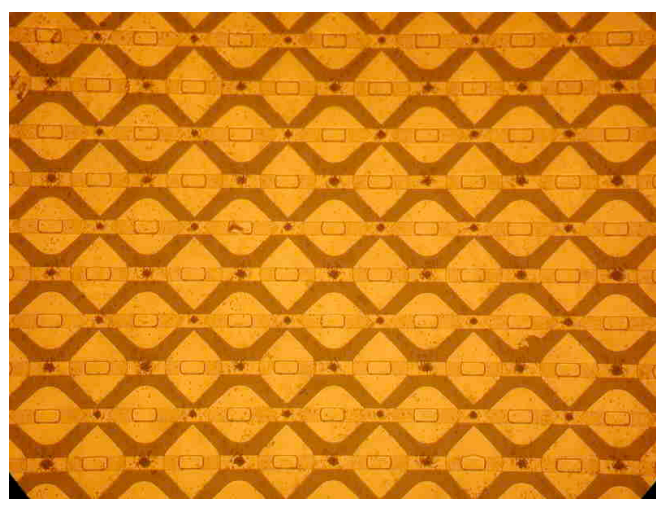

Figure 2: Rangement de nanoparticules par diélectrophorèse sur une biopuce

SATIE/BIOMIS, Université de Tokyo, IETR [4]
Remerciements: Ces recherches ont été effectuées grâce au support des collectivités territoriales (Région Bretagne, Département Ille-et-Vilaine, Rennes-Métropole), du CNRS et de la JSPS.

\section{Références:}

1. M. Washizu, "electrostatic manipulation of biological objects in microfabricated structures" Integrated micro-motion systems, Elsevier Publisher, (1990), 417-432

2. G. Gradl, T. Müller, A. Pfennig, S. Shirley, T. Schnelle, G. Führ, " New micro device for single cell analysis, cell sorting and cloning on a chip: the cytocon instrument", in Proc. of microtas 2000; pp 443-446,

3. L. Cui, H. Morgan, « design and fabrication of travelling wave dielectrophoresis structures», J. Micromech. Microeng., 10, (2000) 72-79

4. M. Frénéa, H. Lhermite, B. Le Pioufle, H. Fujita, "design of biochip microelectrode array for cell arrangement" IEEE-EMBS Conference, Madison, 2002

5. M. Gauthier, E. Piat, "behavior of a magnetic micromanipulator of biological objects"IEEE ICRA02, Vol 2, pp 3199-3204, mai 2002

6. A. Tixier-Mita, Y. Mita, K. Cozic, M. Frénéa, B. Le Pioufle, H. Fujita, "To place cells as an array using aspiration technique", MicroTAS'2002, Nara, Japon

7. E. Ostuni, C. S. Chen, D.E. Ingberg, G.M. Whitesides, "selective deposition of proteins and cells in arrays of microwells" Langmuir, 2001, $17,2828-2834$

8. E. Claveril-Tinture, J. Pine, « Extracellular potentials in low density dissociated neuronal cultures », Journal of Neuroscience Methods, Vol. 117, Issue 1, May 2002, pp 13-21

9. M.C.T.Denyer, M. Riehle, S.T. Britland, A. Offenhauser, «Preliminary study on the suitability of a pharmacological bio-assy based on cardiac myocytes cultured over microfabricated ùicroelectrode arrays » Medical and Biological Engineering and Computing, 1998, 36, pp 638644

10. P. Degenaar, B. Le Pioufle, L. Griscom, A. Tixier, Y. Murakami, K. Yokoyama, E. Tamiya, H. Fujita, " A method for micrometer resolution patterning of primary culture neurons for SPM analysis ", Journal of Biochemistry, Vol. 130, pp. 367-376 (2001)

11. B. Le Pioufle, P. Surbled, K.S. Chun, H. Fujita, H. Nagai, Y. Murakami, E. Tamiya, "Attachment of Cells on Microsystems: Application to the Gene Transfection." Transducers '99, Sendai (J), 1999, pp. 768-771. 